Betreffs anderer Factoren (Aenderung des Druckes und der Vertheilung des Blutes, Producte des Muskelstoffwechsels etc.), die noch überdies an der motorischen Acceleration, wenn auch in viel geringerem Maasse, betbeiligt sein könnten, wird auf eine Untersuchung von $\mathrm{J}$ oh a $\mathrm{n}$ s s o n hingewiesen.

Zum Schlusse erfitlle ich eine angenehme Pflicht, indem ich den Herren Dr. H of $\mathrm{mann}$ und Dr. W i ener meinen berzlichsten Dank sage fur ihre freundlicbe Assistenz bei der langwierigen Registrirung der Herzschläge und bei den Operationen.

(Aus dem physiologischen Institut der Universität Strassburg.)

\title{
Zur Physiologie des Labyrinths.
}

IV. Mittheilung.

\section{Die Beziehungen des Grosshirns zum Tonuslabyrinth.}

Theilweise nach Versuchen von Id a H. Hyde.

Mitgetheilt von

\section{J. Rich. Ewald.}

Eine der auffallendsten Erscheinungen, welche Tauben nach. Operationen am Labyrinth darbieten, ist die typische Kopfverdrehung einseitig labyrinthloser Thiere. Sie tritt anfallsweise auf und jeder einzelne Anfall nimmt einen so regelmässigen und typischen Verlauf, dass sich 6 verschiedene Stellungen des Kopfes angeben lassen, welche für diese merkwürdige Bewegung characteristisch sind. Ich habe sie einzeln abgebildet und als Stellung I-VI bezeichnet ${ }^{1}$ ). Die Rolle, welche diese 6 Stellungen bei den

1) Physiologische Untersuchungen über das Endorgan des Nervus Octavus. Wiesbaden 1892. 
Kopfverdrehungen spielen, ist eine doppelte. Erstens bezeiehnen sie in der den Zahlen entsprechenden Reihenfolge den Weg, den der Kopf durchläuft, um in die bei der jedesmaligen Verdrehung erreichten Endstellung zu gelangen. Sie bilden also gewissermaassen die Durchgangsstationen, welche vom Kopf in schneller Folge auf seinem Wege durcheilt werden. Es gelangt z. B. der Kopf immer nur in die Stellung VI - wenn dies die Endstellung der betreffenden Kopfverdrehung ist - indem er die Stellungen I-VI durchläuft. Aber diese ganze Verdrehung wird nicht gleich im Beginn der Epoche der Anfälle ausgefiihrt. Indem sich die Kopfverdrehung ausbildet, wird der Kopf anfänglich während einiger Tage nur bis Stellnng II, dann einige Tage lang nur bis Stellung III u. s. w. gebracht, und so bezeichnen die angegebenen 6 Stellungen auch zugleich die Endstellungen, bis zu welchen sich die Verdrehung allmählich ausbildet. Während der Epoche der Anfälle bilden sich also diese letzteren zu immer stärkerer Kopfverdrehung aus, und nachdem sie eine kürzere oder längere Zeit auf ihrer Höhe verblieben sind, nehmen sie in umgekehrter Reihenfolge der Endstellungen allmählich wieder ab. Dabei braucht nicht immer das Maximum der Störung, wie es die Stellung VI angibt, erreicht zu werden, ja es kann die Verdrehung nur bis Stellung III ausgebildet werden, und schon dann wieder eine Besserung eintreten. Ueberhaupt sind die individuellen Verschiedenheiten in der Ausbildung und im Verlauf der Anfälle sebr in die Augen springend. Aber sie beziehen sich nur auf die Schnelligkeit, mit der sich die Störung ausbildet, auf den Grad, den sie erreicht, auf die Daner der einzelnen Anfälle und auf die Länge der Zeit, während welcher diese Anfälle auftreten. Gewöhnlich pflegt am 6. bis 8. Tage nach der Operation die Epoche der Verdrehungen za beginnen. Sie kann jedoch auch schon am 5. Tage eintreten und kann bis zum 20. auf sich warten lassen. Die Epoche der Anfälle kann 14 Tage oder Jahre lang dauern. Aber trotz aller dieser individuellen Unterschiede ist doch die Gesetzmässigkeit der Erscheinung eine ganz erstaunliche und mich immer von nenem tiberraschende. Bis jetzt babe ich noch kein Thier beobachtet, welches keine Kopfverdrehung gezeigt hätte, kein Thier gesehen, bei dem die Verdrehungen nicht in der durch die angegebenen Stellungen gekennzeichneten Weise abgelaufen wären.

Und gerade diese erstaunliche Gesetzmässigkeit in dem VerE. Pfüger, Archiv f. Physiologie. Bd. 60. 
halten der Kopfverdrehungen macht dieses Symptom ganz besonders geeignet, um an ihm den Verlauf der vom Tonuslabyrinth ausgehenden Störungen zu beohachten. Nicht, dass man die anderen Symptome, wie z. B. das Kopfwackeln nach Plombirung paralleler Kanäle nicht auch zum Studium heranziehen sollte, aber nur die Kopfverdrehung gestattet uns ein fruheres oder späteres Eintreten der Störung, eine geringere oder stärkere Ausbildung derselben, eine längere oder kïrzere Dauer der krankhaften Erseheinung in so einfacher und sicherer Weise zu erkennen, und iberdies allein durch die Beobachtung des sich völlig selbst iberlassenen Thieres. Es wird uns daher bauptsächlich dies Symptom bei der Beurtheilung der Mittel, welche das Thier besitzt, um die Labyrinthstörungen zu compensiren, leiten und uns lehren, welche Beziehungen zwischen den Functionen des Tonuslabyrinths und denen anderer Organe bestehen.

Lange ${ }^{1}$ ) hat bereits in einer von mir angeregten Arbeit nachgewiesen, dass die Kleinhirnstörungen nichts direct mit den Labyrinthstörungen $z u$ thun haben. Beide treten in ibrer eigenthlimlichen Weise auf, und beide werden bis zn einem gewissen Grade wieder compensirt, gleichgiltig, ob das andere Organ bereits zerstört ist oder nicht. Die Störungen lassen sich einander superponiren. Nur ein geringer Unterschied in der Heftigkeit des Auftretens und in der Daner, während der sich die Symptome in urspriinglicher Stärke erhalten, lässt sich bei der Combination der beiden Operationen feststellen. Indirect scheint daher jedes der beiden Organe die allnählich eintretende Compensirung der Störungen des anderen zu erleichtern, ersetzen können sie sich einander nicht.

Hier miissen einige Worte über die Begriffe der Adaption nud der Ersatzerscheinungen, welche ich in meinem Buche ${ }^{2}$ ) aufgestellt habe, eingeschaltet werden. Unter Adaption verstehe ich diejenigen Vornahmen des Thieres, welche den Zweck haben, die eingetretene Störung zu compensiren und bei welchen nur solche Mittel zur Verwendung kommen, welche in gleicher

1) Lange B., In wieweit sind die Symptome, welche nach Zerstörung des Kleinhirns beobachtet werden, auf Verletzangen des Acusticus zurückzufiuhren? Pflüger's Arch. Bd. 50, p. 615.

2) l. c. p. 270 . 
Weise auch von dem normalen Thiere gebraucht werden. Eine blinde Katze benimmt sich kurze Zeit nach Entfernung der Augen in gleicher Weise wie eine sehende, wenn diese vorubergebend ins Dunkle gebracht wird. Sie bewegt sich vorsichtig und orientirt sich so gut es eben ihr Ortssinn, ihr Gehör, ihr Geruch und Getast ohne besondere Uebung und Ausbildung: zulassen. Sie adaptirt sich an deu Mangel des Gesichts. Aber sie folgt nicht mehr wie früher dem Diener durch alle Räume des Instituts, sie springt nicht mehr nach dem auf den Boden geworfenen Fleischstück. Beides hat sie bisher nur mit Hilfe des Gesichtssinnes ausführen können, und sie muss nun erst lernen auf die betreffenden Geräusche zu achten, welche es ihr ermöglichen, bei diesen Handlungen den Mangel der Augen zu compensiren. So entstehen Ersatzerscheinungen. Sie werden erst nach dem Verlust der normalen Functionen ausgebildet und gewissermaassen von dem Thier erlernt. Sie können übrigens auch negativer Natur sein: ursprünglich läuft die geblendete Katze, wenn man sie erschreckt und jagt, wild gegen ein Hinderniss an, in späterer Zeit wird sie sich in einem ihr nicht bekannten Raume lieber hinkanern und zur Wehr setzen, als blindlings davon eilen. Ein sehr characteristisches Beispiel bietet der Hund mit durchschnittenem Lendenmark, welcher allmählich wieder lernt, mit horizontalem Riicken durch das Zimmer zu gehen. In den ersten Wochen nach der Operation schleift er seinen Hinterkörper anf dem Boden nach, so dass der Rúcken nach abwärts gerichtet ist und die Sehwanzwurzel den Boden berührt. Allmählich lernt er aber immer mehr, seine ganze Körperlast durch die Vorderbeine zu stultzen. Die Brust- und Vorderbeinmuskulatur erstarkt und er balancirt endlich beim Gehen durch das Zimmer den ganzen Körper mit beständig horizontal gehaltenem Rücken allein auf den Vorderbeineu. Die Hinterbeine hängen von dem Becken herab und berubbren mit den Zehen den Boden. Die hierdurch ausgelösten Reize bewirken ein reflectorisches Pendeln der Hinterbeine, und so werden die Gehbewegungen des Thieres denen des normalen Hundes in merkwlirdiger Weise wieder äbnlich. Thatsächlich ist dabei das Symptom des abwärts gerichteten Ruekens, welches aach der Durchschneidung des Lendenmarkes auftrat, durch eine Ersatzerschein ung völlig ausgeglichen worden.

Ich möchte ïbrigens, die Begriffe der Adaptions- und Ersatz- 
erscheinungen auch auf die unserer Beobachtung nicht direct zugänglichen Vorgänge innerhalb des Körpers, speciell im Nervensystem, ausdehnen. Die Ausbildung neuer nervöser Leitungsbahnen würde z. B. eine Ersatzerscheinung sein.

Kebren wir zu den Tauben mit einseitigem Lảbyrinthmangel zurlick und fragen wir, weshalb die Kopfverdrehung nicht unmittelbar nach der Operation auftritt, und weshalb sie nach einer mehr weniger langen Epoche ihres Bestehens schliesslich wieder verschwindet.

Dass der Functionsausfall des Tonuslabyrinthes nicht sofort seine ganze Wirkung ausübt, sondern durch einen nur allmäblich abnehmenden Reizzustand des Octavusstammes selbst (Pseudofunction) theilweise ausgeglichen wird, geht aus der Beobachtung der Thiere direct hervor. Aber warum verdrehen sie nicht sofort nach der Operation den Kopf ein wenig, da ja ein gewisser Functionsausfall des Tonuslabyrinths sofort zu constatiren ist, und warum wird der Kopf nicht später mit Abnahme der Pseudofunction beständig immer mehr und mehr bis zur Stellung VI verdreht. Hier handelt es sich um eine Adaptionserscheinung. Wenn man eine Taube daran gewöhnt hat, ein auf dem Kopfe befestigtes leichtes Stäbchen zu tragen, so kann man ein kleines Gewicht einseitig an demselben befestigen und dieses allmählich nicht unbedentend vergrössern, ohne dass dadurch die Taube zu einer schiefen Haltung des Kopfes veranlasst würde. Sie pickt auch mit dieser einseitigen Belastung des Kopfes Erbsen anf und kann olne irgend welche Störung fliegen. Und doch müssen alle Muskeln, die den Kopf und den Hals bewegen, in ganz besonderer Weise zusammenwirken, um die einseitige Belastung zu compensiren. Wir beobachten dasselbe zuweilen auf der Strasse, wenn ein Pudel seinem Herrn den Schirm oder Stock nachträgt, und denselben nicht ganz in der Mitte gepackt hat. Er hält deswegen den Kopf doch nicht schief. Die vorbandenen und dem Thiere normaler Weise zu Gebote stehenden Innervationsmittel gleichen die Asymmetrie der Belastung aus. Die Gradhaltung des Kopfes ist unter diesen Umständen eine Adaptionserscheinung, welche erst bei einem Uebermaass der einseitigen Belastung versagt. Sie versagt auch, wenn das Thier aufgeregt ist, oder wenn es eine besondere direct vom Willen dictirte Bewegung mit dem Kopf auszufibren beabsichtigt, wo denn offenbar die Willensimpulse nicht in derselben Weise 
asymmetrisch wie es zur Compensirung nöthig wäre und wie es bei den reflectorischen Bewegungen thatsächlich geschieht, zu den Muskeln gelangen. So erklärt sich auch das anfallsweise Auftreten der Kopfverdrehung und die Unfähigkeit des Thieres, die Störung durch einen besonderen Willensact wieder zu beseitigen. Der Anfall geht eben nur voriber, wenn das Thier aufhört eine Bewegung des Kopfes zu intendiren, sich gewissermaassen ganz harmlos sich selbst überlässt.

Das Aufhören der Kopfverdrehungen möchte ich dagegen als Ersatzerscheinung auffassen, indem ich annehme, dass sich allmählich die Art der Innervation auch fiür die willkürlichen Muskelbewegungen ändert, so dass die fehlende Einwirkung des Tonuslabyrinthes auch bei willkürlichen Anstrengungen ausgeglichen wird. Um einen solchen Ersatz herbeizuführen, müssen Einrichtungen getroffen werden, welche im normalen Thiere nicht vorhanden sind, und wir können füglich fragen, von welchen Theilen des Körpers diese practische Regulirung und Umformung der Functionen ansgebt.

Es ist in dieser Beziebang zunächst wichtig, zur Gewinnung eines einheitlichen Standpunctes, uns einen kurzen Ueberblick iiber den Ersatz des Tonuslabyrinths bei den verschiedenen Wirbelthieren zu verschaffen. Die einseitig labyrinthlosen Frös e h e zeigen unmittelbar nach der Operation bereits die Kopfverdrehung in demselben Grade, den sie überhaupt erreicht. Es bängt dies offenbar damit zusammen, dass bei den Kaltblütern keine Reizung des Octavusstammes durch die Operation gesetzt wird, und daher in Folge des Mangels einer Pseudofunction die Störung gleich im ganzen Umfange auftritt. Die Adaptionserseheinungen werden daher nicht durch späteres Anwachsen der Störung unzureichend. Es werden auch keine Anfälle beobachtet. Erhält man die Thiere lange Zeit am Leben, so zeigen sie auch nach einem Jahre noch dieselbe characteristische Haltung des Körpers wie unmittelbar nach der Operation. Legt man sie auf den Rucken, und kehren sie sich dann in die normale Lage zuriuck, so bleibt auch nach dieser langen Zeit die gekreuzte Hinterpfote häufig. in gestreckter Lage liegen und wird nicht an den Körper herangezogen. Eine Abschwächung der Symptome ist nur in ganz geringem Maasse zu bemerken. Ersatzerscheinungen sind also so gut wie nicht vorhanden. 
Bei den einseitig labyrintblosen $\mathrm{T}$ a $\mathrm{u}$ be $\mathrm{n}$ bilden sich die Ersatzerscheinungen nur ganz langsam, und nie sehr vollständig aus. Gewöhnlich ist erst nach vielen Wochen die Epoche der Kopfverdrehungen beendet. Es dauert zuweilen Monate, bis die Thiere wieder in die Höhe fliegen können. Wie oben bereits erwähnt, machen sich individuelle Verschiedenheiten dabei sebr bemerkbar, und so scheint es mir nicht ausgeschlossen, dass sich einmal bei einem Thiere die Kopfverdrehung nur ganz rudimentär ausbilden könnte, weil die Ersatzerscheinungen zu schnell eintreten, oder dass bei einem anderen Thiere die betreffenden Anfälle aus dem umgekehrten Grunde nie ganz verschwinden.

Bei den $\mathrm{Hunden}$, denen man ein Labyrinth vollständig zerstört hat, gehen die Erscheinungen im Verlauf von wonigen Monaten so vollständig zurück, dass sich das Thier nur bei Anwendang feinster Prïfungen von einem normalen unterscheiden lässt.

Endlich müssen wir für den $\mathrm{M}$ e $\mathrm{n} \mathrm{s} \mathrm{ch}$ e $\mathrm{n}$ einen noch vollständigeren Ersatz, als wir ihn bei den Hunden kennen gelernt haben, annehmen, da nach der allmählichen Zerstörung sogar beider Labyrinthe, oder für den Fall, dass dieselben von Jugend auf functionsunfähig waren, nur ganz unbedeutende Störungen beobachtet werden können. Frtiher hat man von diesen gar nichts gewusst, und erst in letzter Zeit haben die interessanten Arbeiten von James ${ }^{1}$ ), A lois $\mathrm{Kreidl^{2 }}$ ), Pollak ${ }^{3}$ ) u. A. einige Symptome von dem Functionsausfall des Tonuslabyrinths am Menschen aufgedeckt. $\mathrm{Zu}$ diesen mögen bei noch genauerer Untersuchung der Taubstummen noch einige bisher unbeachtete Störungen hinzukommen, es wird aber immer richtig bleiben, dass die Ersatzerscheinungen beim Menschen den höchsten Grad ibrer Ausbildung erreichen.

Eine ähnliche Verschiedenheit in der Ausbildung von Ersatz-

1) James, W., The sense of dizziness in deaf-muter. Amer. Journ. of Otology 4. 1882.

2) Kreidl, A., Beiträge zur Physiologie des Ohrlabyrinths auf Grund von Versuchen an Taubstummen. Pflüger's Arch. Bd.51. p. 119.

Derselbe. Zur Lehre vom Gleichgewichtsorgan. Centralbl. f. Physiologie. 1893. p. 165.

3) Pollak, J., Ueber den galvanischen Schwindel bei Taubstummen a. s. w. - Pflüger's Arch. Bd. 54, p. 188. 
erscheinungen trefien wir nach der Fortnahme der Augen an. Der geblendete Froseh ist vollkommen hülflos und verhält sich in vieler Beziehung zum Verwechseln ähnlich wie ein grosshirnloser. Die Anregungen, welche das Thier durch den Sehact unter normalen Verhältnissen erhält, sind so wichtig für alle Vornahmen des Thieres und werden so wenig durch andre Anregungen nach der Blendung ergänzt, dass die Störung eine erstaunlich grosse ist. Es konnte daher das Verhalten des geblendeten Frosches zu dem Irrthum führen, der Goltz'sche Quackversuch berube auf Blind. heit des Thieres. In der That gelingt der Versuch nach Fortnahme des Auges fast ebensogut, wie nach Entfernung des Grosshirns.

Die blinde Taube bat Schrader ${ }^{1}$ ) in musterhafter Weise beschrieben. Wir sind erstaunt, in nur so geringem Umfange die Ersatzerscheinungen auftreten zu sehen. Es fallen nicht nur diejenigen Handlungen des Thieres fort, bei denen das Sehen eine nothwendige Bedingung ist, sondern in hohem Grade auch noch alle diejenigen Lebensäusserungen, welche zwar ohne den Sehakt ausgefübrt werden können, zu denen aber ein Gesichtseindruck die einleitende Veranlassung zu geben pflegt. Die Taube bewegt sich wenig, findet sich in ihrem Verschlag schlecht zureeht, nimmt nur Nahrung auf, wenn sie durch excessiven Hunger dazu getrieben wird. Sie kümmert sich nicht um ihre Genossen, der Geschlechts. trieb scheint erloschen, kurz, wie auch Seh rader richtig bemerkt, die blinde Taube ähnelt noch in vieler Beziebung dem grosshirnlosen Thiere, wenn wir auch bei den Tauben schon im Stande sind, sehr deutliche Unterschiede zwischen den Folgen beider Eingriffe festzustellen.

Ganz im Gegensatz zur blinden Taube imponirt uns am blinden Hunde, und noch mehr an der blinden Katze, wie vorzüglich der Mangel des Gesichtes ergänzt wird. Die blinde Katze weiss schliesslich im Hause Bescheid wie die sehende. Sie begattet sich und zieht die Jungen auf, sie fängt Mäuse und springt auf das zu Boden geworfene Stück Fleisch mit ganz überraschender Sicherheit. Alle anderen Sinne werden herangezogen, um das fehlende Auge zu ersetzen, und wäre nicht der Gesichtssinn für

1) Schrader, M., Zur Physiologie des Vogelgehirns. Pflüger's Arehiv. Bd. 44. p. 175. 
viele Dinge unersetzlich, so könnten wir das operirte Thier wohl nur schwer von dem normalen unterscheiden.

Die Verhältnisse beim blinden Menschen mit der ausserordentlich bohen Ausbildung der Ersatzerscheinungen sind bekannt. Wir brauchen hier fuglich nicht darauf einzugehen.

Es erscheint nun als höchst bemerkenswerth, dass ein enger Zusammenhang zwischen der Nothwendigkeit des Sehactes für das Zustandekommen der normalen Lebensäusserungen einerseits, und der Bedeutung des Grosshirns für das Sehen andrerseits zu bestehen scheint. Wir können nämlich sagen: Je weniger Einfluss das Grosshirn auf das Auge hat, desto grösser ist die durch Fortnahme der Augen verursachte Störung im Verhalten des betreffenden Thieres. Nimmt man daher an, dass auch die übrigen Grosshirncentren mit der Ausbildung des Sehcentrums etwa gleichen Schritt halten, so lässt sich der Satz anfstellen: Wabrscheinlich werden die Ersatzerscheinungen für das Auge von den Grosshirncentren ausgebildet. Beim Froseh hat man bisher kein Sehcentrum gefunden, und der geblendete Frosch ist auch völlig hüllfos. Bei den Tauben scheint mir ein Sehcentrum erwiesen zu sein. Wenn es auch andrerseits durch die Schrader'schen Untersuchungen feststeht, dass die grosshirnlose Taube noch ihre Gesichtsempfindungen verwerthen kann (ein Befund, den wir in Folgendem zu bestätigen Gelegenheit finden werden). Beide Angaben lassen sich aber sehr wohl mit einander in Einklang bringen. Das Sehcentrum wird nicht plötzlich in der Thierreihe von einem Gehirntheil zum anderen verschoben worden sein. Das nicht im Grosshirn gelegene Sehcentrum ist offenbar bei der Taube noch functionsfähig, aber gleichzeitig können doch bercits die Centren im Grosshirn eine wichtige Rolle beim Sehact spielen. Beim Hunde wird das Sehen schon durch die Fortnahme relativ kleiner Theile des Grosshirns sehr beeinträchtigt. Trotzdem sind auch bei ihm, wie die Goltz'schen Versuche lehren, die ausserhalb des Grosshirns gelegenen Centren noch nicht völlig zu Grunde gegangen. Was endlich den Menschen betrifft, so ist die Abhängigkeit des Sehactes vom Grosshirn bei ihm eine noch viel grössere als beim Hunde.

Ist nun das Eintreten der Ersatzerscheinungen nach Fortnahme eines Sinnesorganes von der Ausbildung der Grosshirncentren, welche die nicht geschädigten Sinnesorgane versorgen, 
abhängig, so müssten also auch die Ersatzerscheinungen für das Tonuslabyrinth durch die Grosshirncentren ausgebildet werden. Diese Annahme erscheint sehr verlockend, und es kann uns nicht gegen sie einnehmen, dass das Tonuslabyrinth nur indirect und in gewisser Beziehung als Sinnesorgan (Goltz'scher Sinn und Otolithenapparat) functionirt. Denn alles was wir bisher von den Sinnesorganen angefiibrt haben, bezieht sich in gleicher Weise auch auf die Bewegungen, mag man die betreffenden Grosshirncentren nun als motorische oder sensible ansprechen.

Dies ist der Gedankengang, der mich veranlasst hat, bei den verschiedenen Thieren Versuchsreihen anzustellen und anstellen zu lassen iiber die Abhängigkeit der Ersatzerscheinungen nach Fortnahme oder Verletzung der Labyrinthe von der Ausbildung der Grosshirncentren. Die Versuche am Froseh habe ich schon seit Jängerer Zeit abgeschlossen. Hier liegen die Verhältnisse sehr einfach und sind schon von Schrader ${ }^{1}$ ) in gewisser Beziehung klar gelegt worden. Doch hat Schrader nicht genïgend lange Zeit Thiere am Leben erhalten, von denen gleich bei der ersten Operation die einen nur das Labyrinth, die anderen Labyrinth und Gehirn verloren hatten. Nur durch viele solche Vergleichsthiere, welche sehr lange am Leben geblieben sind, kann man ein sicheres Urtheil uber die geringen sich zeigenden Unterschiede gewinnen. Schrader constatirte, dass bei labyrinthlosen Fröschen durch Fortnabme des ganzen Grosshirns die Labyrinthsymptome nicht beeinflusst werden. Er zeigte damit, dass die Störungen der beiden Organe nicht direct von einander abhängig sind und beim Frosch superponirt werden können, wie Lang $\mathrm{e}^{2}$ ) dies in Beziehung auf das Kleinhirn und das Labyrinth bei den Tauben nachgewiesen hat. Ueber die geringe Besserung der Symptome, welche nach langer Zeit auch bei den Fröschen eintritt, hat Schrader aus oben erwähntem Grunde keine ausreichenden Beobachtungen angestellt, und so musste ich zum Vergleich einseitig und doppeltseitig labyrinthlose Frösche, von denen ein Theil zu gleicher Zeit entgrosshirnt worden war, viele Monate lang am Leben erhalten. Danach zeigte es sich in der That, dass schliess-

1) Schrader, M., Zur Physiologie des Froschgehirns. Pflüger's Archiv Bd. 41 p. 75.

2) 1. c. 
lich, meist erst am Ende der Beobachtungszeit, die Grosshirn besitzenden Frösche im Gebrauch der Muskulatur ihren grosshirnlosen Genossen überlegen waren. Der Sprung ist bei den ersteren weniger steil und weniger schief nach der Seite hin gerichtet. Beim Herabkommen auf den Boden fallen sie etwas weniger ungliicklich auf. Die Schwimmbewegungen erfolgen häufiger mit beiden Beinen gleichzeitig, während die grosshirnlosen Thiere die hinteren Extremitäten nur alternirend bewegen. Aehnlich hat sich auch bei den einseitig labyrinthlosen Thieren ein geringer Unterschied entwickelt. Er lässt sich aber schwer in Worten ausdrücken, und man kann nur sagen, dass bei den Grosshirn besitzenden Fröschen die Störungen sämmtlich an Intensität etwas mebr abgenommen haben. Ueberhanpt ist aber der Untersehied, den der Besitz des Grosshirns hervorbringt, nur ein sehr geringer, und wir gelangen also zu dem Resultat: Fbenso gering, wie die Ausbildung von Ersatzerscheinungen bei den labyrinthlosen Fröschen ist, ebenso unbedeutend ist auch der Einfluss, den die Entfernung des Grosshirns auf die Stärke der Labyrinthsymptome hat.

Dieselbe Frage an Tauben zu behandeln übertrug ich Fräulein Ida H. Hyde, deren Versuche und Resultate in Folgendem geschildert werden sollen. Es wurde zunächst eine Anzahl von etwa 10 grosshirnlosen Tauben hergestellt, was bekanntlich, wenn man auf vollständige Entfernung des Grosshirns bält, keine ganz einfache Aufgabe ist, da sehr viele Thiere die Operation nicht iaberstehen. Immerhin waren $\mathrm{H}$ y de's Resultate in Bezug auf das Ueberleben der Thiere giunstiger als diejenigen ihrer Vorgänger, welche hier in Betracht kommen. $\mathrm{M} \mathrm{ank}^{1}$ ) erhielt $80 \%$ Todesfälle; Schrader $\left.{ }^{2}\right) 75 \%$. Munk hat es wahrscheinlich gemacht, dass die älteren Autoren wohl meist nur unvollständige Entfernungen des Grosshirns herbeigefuhrt baben. Sie wurden, wie mir scheint, zum Stehenlassen von grösseren Resten durch ungiunstige Operationsbedingungen veranlasst. Man muss sich vor allen Dingen bei der Operation genïgenden Platz durch Fortnahme des Sehädel-

1) Munk, H., Ueber die centralen Organe für das Sehen und das Hören bei den Wirbelthieren. Sitzungsber. d. Berliner Academie. 1883. p. 793 u. 1884, p. 549.

2) Zur Physiologie des Vogelgehirns 1. c. 
dachs in möglichst grosser Ausdehnung verschaffen. Munk ist in dieser Beziehung wenig ängstlich vorgegangen, lässt aber doch noch in der Medianlinie eine Knochenspange uber dem grossen Sinus zwischen den beiden Hirnhälften stehen und nimmt dann die Hemisphären von binten und der Seite her fort. Die Knochensubstanz bröckelt er langsam Stückehen für Stuickchen mit einer Pincette $a b$, wobei er nach vorn, hinten und der Seite (unten) so weit geht, wie man iberhaupt rationeller Weise gehen kann. Schrader und $\mathrm{Hyde}^{1}$ ) sind ihm in dieser letzteren Beziehung gefolgt. Man geht nach vorne so weit, bis man der Spitzen der Riechlappen ansichtig wird. Hierzu ist es nöthig, keilförmig nach vorne noch ein Stïck weit zwischen die Orbitalränder vorzudringen. An dieser Stelle sind die beiden Schädeltafeln bereits um 1-2 mm von einander durch spongiöse Knochenmasse getrennt. Von der vordersten Spitze läuft dann der Knochendefect jederseits an dem Orbitalrand entlang, bis er scharf nach hinten umbiegend einer Linie folgt, welche man sich von dem Mittelpunkte der Cornea zur Linea semicircularis (Ansatz der Nackenmuskulatur) gezogen denken kann. Den hinteren Rand des Defectes bildet die Grenze zwischen Grosshirn und Kleinhirn, aber anch hier sind die beiden Knochentafeln schon beträchtlich vou einander entfernt und ihr Zwischenraum mit spongiöser Masse angefïllt.

Schrader ist noch etwas weiter gegangen als $M u n k, d . h$. er hat sich noch mehr Platz für die Operation geschafft, indem er es unterliess, eine mediane Knochenbrücke zwischen vorne und hinten stehen zu lassen. Da eine solche Brücke nur beim Operiren binderlich ist, den Heilprocess unglinstig beeinflusst und in keiner Weise nützt, so ist dies Vorgehen zu billigen. Auch Hyde hat das ganze Schädeldach entfernt. Munk schont ferner den Sinus, seinen Bluterguss fürchtend. Schrader schneidet ihn hinten durch, torquirt ihn und schlägt ihn nach vorne zuritek. Hyde hat ihn vorn und hinten durchgeschnitten und sich um ihn weiter nicht bekümmert, denn von ihm geht die Gefahr der Blutung nicht aus. Munk und Schrader haben dann mit Stäbchen die Hemis-

1) Statt der Pincette benutzte $\mathrm{Hyde}$ eine flache Zange, mit der man leicht zwischen Knochen und Dura, ohne letztere $z u$ verletzen, vordringen kann. Die Entfernung des Schädeldaches gelingt auf diese Weise sehr viel schneller als bei Gebrauch einer Pincette. 
phären von hinten her von ibrer Unterlage abgehoben und die Pedunculi - Munk mit dem Stäbchen, Schrader mit einem Messer - durchtrennt. Darauf lässt sich das gesammte Grosshirn in toto aus dem Schädel herausnehmen, und es werden dadurch die Lobi optici, die Thalami optici, das Chiasma nerv. opt. und die Stimpfe der Pedunculi in der Schädelhöhle sichtbar. Hyde verfuhr bei der Herausnahme der Hemisphären etwas an. ders. Nachdem sie die Dura mit dem Sinus entfernt batte, gebrauchte sie zur Herausnahme der Hemisphären einen grossen Excavator - seine runde Platte hat $5 \mathrm{~mm}$ Durchmesser -, welcher wohl auch durch einen ganz flachen runden Löffel von derselben Grösse, der beinahe rechtwinklig rom Stiel abgebogen ist, ersetzt werden könnte. Mit diesem Instrumente werden die Hemisphären von hinten her aufgehoben, nach vorn gewälzt und von den Pedunculis abgerissen. Die Praxis hat dabei ergeben, dass der stumpfe Löffel das Chiasma nicht verletzt und dass nicht leicht irgend welche Reste des Grosshirns zurïckbleiben. Im Wesentlichen handelt es sich also um die Munk'sche Methode, das Grosshirn in toto von der Unterlage abzuheben. Das Gesichtsfeld soll bei der Operation immer klar bleiben. Ist die Blutung dennoch so gross, dass sie einen freien Ueberblick verhindert, so pflege ich den Anblick der freigelegten Fläche durch einen constanten kalten Wasserstrahl zu ermöglichen. Hyde hat in den meisten Fällen die Blutung mit warmer, physiologischer Kochsalzlösung zum Stehen gebracht, eine Methode, die ich selbst bei der Enthirnung nie angewendet habe und von deren Ueberlegenheit uber andere Methoden ich mir auch durch die Hyde'schen Versuche noch kein endgiltiges Urtheil habe bilden können. Der Hautschnitt wurde stets in der Medianlinie ausgeführt, was offenbar wegen der geringeren Blutung besser ist, als ihn nach Schrader in die Richtung von Ohr zu Ohr zu verlegen. Die Wunde wurde nach Stillung der Blutung sorgfältig vernäht, ohne einen Wattetampon in der Schädelhöble zu lassen. Nach völliger Beendigung der Operation ${ }^{1}$ ) schlug man um Fligel und Brust einen Gaselappen, der durch eine Nadel zusammengehalten wurde. Es hindert dies die Tauben sich zu be-

1) Während der ganzen Operation befindet sich die Taube in meinem Taubenhalter, der durch Fixirung des Kopfes das Operiren wesentlich erleichtert. Auch Schrader hat stets den Taubenhalter benutzt. 
wegen, und so verblieben sie einige Tage lang. Hy de fand auch, dass es vortheilhaft für das Befinden der Thiere sei, gekochte Erbsen zu füttern, ferner gab sie stets einige Kieselsteinchen zum Futter.

Wie schon oben bemerkt, war die Sterblichkeit der so operirten Tauben eine relativ geringe, etwa $50 \%$. Wenn die Thiere die Operation 10 Tage bis einige Wochen gut überstanden batten, so führte $\mathrm{Hyde}$ nun an ihnen die Labyrintboperation aus. Auf die Symptome, welche die grosshirnlosen Thiere vor der Labyrinth. operation darboten, soll hier, da die Enthirnung ja zu einem besonderen Zwecke vorgenommen wurde, nicht näher eingegangen werden. Nur sei erwähnt, dass stets darauf geachtet wurde, ob die Thiere noch auf Licht und auf Schall reagirten. Letzteres war immer der Fall, ersteres konnte bei zwei Tauben, welche sich iberhaupt sehr ungern bewegten, nicht nachgewiesen werden. Die Sectionen, welche theilweise von $\mathrm{Hyde}$, theilweise von mir ausgeführt wurden, ergaben sämmtlich ${ }^{1}$ ) vollständigen Verlust des Grosshirns, wie es bei der geschilderten Operationsmethode auch kaum anders möglich ist. Bei einigen Thieren war ansser dem Grosshirn noch ein kleiner Theil der Thalami optici zu Grunde gegangen, ob nachträglich oder schon bei der Operation konnte nicht mit Sicherheit festgestellt werden. Hyde war also in der Lage, gewissermaassen als Nebenresultat ihrer Versuche die Sc hrader'sche Angabe, dass grosshirnlose Tauben noch auf Licht reagiren, den $M \mathfrak{u} k$ 'schen Behauptungen gegenüber bestätigen zu können.

Den geschickten Händen Hyde's und ihren an Präparirlubungen und Lupenarbeiten gewöhnten Augen, war es ein Leichtes von mir die Methodik der hier in Betracht kommenden Labyrinthoperationen zu erlernen, so dass sie nach einer kurzen Lehrzeit die Operationen vollkommen selbständig ausführte. Sie plombirte einen oder beide Canales externi, um das horizontale Kopfwackeln zu erzeugen; oder sie plombirte den Canalis anterior auf der einen und den Canalis posterior auf der anderen Seite (also

1) Bei dieser Angabe wird von einer Taube abgesehen, welche schon eine Woche nach der Operation spontan hoch aufflog und hierdurch verdächtig wurde. Hyde fand bei der Section, dass ein Stückchen Grosshirn erhalten war. 
immer die functionell zusammengehörigen Canäle), um das diagonale Kopfwackeln entstehen zu lassen. Endlich nahm Hyde auch einseitig oder doppelseitig das gesammte Labyrinth heraus, wobei ich immer als Bedingung stellte, dass die 5 Octavusstimpfe gesehen und mit einem feinen Häckchen hin- und herbewegt werden mussten,

Was nun die Resultate, zu denen Hyde gekommen ist, anbelangt, so können dieselben nicht mit ähnlichen Operationen fruberer Autoren verglichen werden. Es ist unwahrscheinlich, wie M u n k richtig hervorhebt, dass die friberen Gehirnoperationen vollständig ausgefuhrt wurden, zugleich ist es aber ganz sicher, dass die Labyrinthoperationen unbrauchbar waren. Die Methodik für die Fortnahme des ganzen Organs wie für die Verletzungen desselben war noch nicht ausgebildet, und grade beim Labyrinth kommt so viel auf die Ausfuhrung der Operation an. Denn einerseits kann durch den Ausfluss der Bogenflïssigkeiten das gesammte Organ anormal werden, andrerseits werden kleine zurlickbleibende Reste bäufig abgekapselt, und wenn sie mit einem der Stämme noch in Verbindung stehen, so bewirken sie normale oder anormale Restfunctionen. Uebrigens baben sich die frtiheren Autoren Flourens ${ }^{1}$ ), Berth old ${ }^{2}$ ), B ernhard ${ }^{3}$ ), Loevenberg ${ }^{4}$ ), $\mathrm{Cy}$ on $^{5}$ ) meist darauf beschränkt, zu zeigen, dass die ihnen bekannt gewesenen Labyrinthstörungen (ein Gemisch von Ausfalls- und Reizerscheinungen) anch nach den von ihnen ausgeführten Gehirnzerstörungen bestehen blieben, und dass daber die Labyrinthstörungen nicht nur der Ansdruck psychischer Vorgänge sein können. Es ist dies eine Thatsache, die wir hente allgemein an-

1) Flourens, Nouvelles expériences sur l'indépendance respective des fonctions cérébrales. Comptes Rendus des Séances de l'Acad. des sciences T. 52. p. 673.

2) Berthold, E., Ueber die Function der Bogengänge des Ohrlabyrinths. Arch. f. Ohrenheilkunde. IX. N. F. III. p. 77.

3) Bernhardt, A, Experim. Beiträge zur Physiologie d. Bogengänge d. Ohrlabyrinths. Pflüger's Arch. Bd. 12. p. 471.

4) Loevenberg, Ueber die nach Durchschneidung der Bogengänge des Ohrlabyrinths auftretenden Bewegungsstörungen. Arch. f. Augen- u. Ohrenheilkunde. Bd, 3. p. 1.

5) Cyon, E., Ueber die Function der halbzirkelförmigen Kanäle. Pflüger's Arch. Bd. 8. p. 306. 
erkennen und aus der Art der Störungen selbst mit Sicherbeit erschliessen.

Durch die vorherige Fortnahme des Gebirns werden die Labyrinthstörungen qualitativ nicht geändert. Die Plombirung der horizontalen Canäle bewirkt auch bei den grosshirnlosen Thieren horizontales Kopfwackeln und Mangel der Reactionsbewegungen bei horizontaler passiver Rotation, falls gleichzeitig die Gesichtseindricke ausgeschlossen sind. Dasselbe gilt auch für die anderen Kanalpaare, nur dass es sich natiurlich bei ihnen um die ihrer Lage entsprechende Ebene handelt. Nach einseitiger Fortnahme des ganzen Labyrinths tritt die typische Kopfverdrehung ein. Nach doppelseitiger Fortnahme wird der Kopf zwar grade gehalten, sinkt aber bei Belastung und ebenso im Dunkeln, falls er nicht gestiutat wird, der Schwere folgend herab u. s.w. Es genigt hier diese wenigen am meisten in die Augen springenden Symptome anzuführen. Die tabrigen feineren Veränderungen waren aber ebenfalls leicht nachweisbar. Im Grossen und Ganzen traten die Störungen etwas stärker als bei den Grosshirn besitzenden Thieren auf und entwickelten sich schneller. Besonders deutlich war dies bei der Kopfverdrehung zu beobachten. Sie erreichte fast bei allen Thieren Stellung VI. Bei einer Taube wurde bereits am folgenden Tage nach der Operation der Kopf in Stellung VI gehalten. Es lässt dies auf eine herabgesetzte Adaptionsfähigkeit schliessen, und da die Adaption rein reflectorisch zu Stande kommt, und die Reflexe an hirnlosen Thieren prompter abzulaufen pflegen, so erseheint dieses Resultat zunächst sehr auffallend. Bedenkt man aber, dass auch die Empfindungsorgane, durch welche die reflectorische Adaption zu Stande zu kommen scheint, durch die Entgrosshirnung geschädigt worden sind, so wird dadurch die geringere Adaption verstaindlich, ja nothwendige Folge des Grosshirnmangels.

Aber dasjenige Resultat der Hyd e'schen Beobachtungen, auf welches das Hauptgewicht zu legen ist, liegt in der geringen Ausbildung der Ersatzersebeinungen bei den grosshirnlosen Thieren. Die Epoche der Kopfverdrehungen dehnte sich bei allen einseitig labyrinthlosen Tauben libermässig - und zwar bis zu ihrem Tode - aus. Bei einem Thiere wurde die Kopfverdrehung zur da u e r $\mathbf{n}$ den Stellung, die auch nach 6 Monaten niemals aufgegeben wurde. Keine der einseitig operirten Tauben lernte wieder fliegen, keine 
der doppelt operirten Tauben wieder selbständig essen oder trinken. Das Kopfwackeln nach den Plombirungen besserte sich nur sehr langsam und konnte aucb nach vielen Wocben durch geringe Reize fast in ursprünglicher Stärke wieder hervorgerufen werden. Wir unterlassen es, eine Bescbreibung jedes einzelnen Thieres und jedes einzelnen der vielen merkwiirdigen Symptome (vergl. Cap. I, II und VI meines Buches über den Nervus Octavus) zu geben. Es wirde damit nichts erreicht werden, da sich jedes Symptom im Einzelfalle auch bei einer grosshirnbesitzenden Taube ähnlich stark entwickeln und auch einmal besonders lange erhalten kann. Eine Vergleichung der Gesammterscheinungen lässt aber den geschilderten Einfluss des Grosshirns in unverkennbarer Weise hervortreten.

So ergab sich denn für die Taube: Der geringen Ausbildung ibrer Grosshirncentren entsprechend, werden auch die Ersatzerscheinungen für die Labyrinth. symptome nach Fortuahme des Grosshirns zwar in recht deutlicher. Weise, immerbin doeb nur in geringem Umfange vermindert. Die Hyde'schen Tauben bestärken uns daher in der Vermuthung, es möchten die Grosshirncentren bei der Ausbildung der Ersatzerscheinungen eine wichtige Rolle spielen. Freilich bleibt dies vorläufig noch eine Vermuthung, welche erst als bewiesen betrachtet werden kann, falls bei den Hunden die sonst so stark ausgebildeten Ersatzerseheinungen nach Fortnahme gewisser Gehirncentren ausbleiben werden. Sollte uns dieser Beweis glïcken, so würden wir durch die Kienntniss, von welchen Gehirntheilen die Ersatzerscheinungen ausgebildet werden, zu gleicher Zeit sowohl uber die Labyrinthstörungen wie tiber die Grosshirnfunctionen einen neuen Aufscbluss erhalten. 